\title{
Determination of Monomer Reactivity Ratios in Copolymerization from a Single Sample and Its Application to the Acrylonitrile-Methyl Methacrylate System
}

\author{
Riichirô Chûjô, Hisashi UbARA, ${ }^{*}$ and Atsuo Nishioka \\ Department of Polymer Engineering, Tokyo Institute of Technology, \\ 12-1 Ookayama 2-chome, Meguro-ku, Tokyo 152, Japan.
}

(Received November 11, 1971)

\begin{abstract}
Monomer reactivity ratios $r_{1}$ and $r_{2}$ can be determined from a single sample of a copolymer if the mole fraction of each dyad is given. Similar treatment is possible for ratios $r_{11}, r_{12}, r_{21}$, and $r_{22}$ if the mole fraction of each triad is given. According to this method of determination of monomer reactivity ratios $r_{12}$ and $r_{22}$ were obtained for acrylonitrile $\left(\mathrm{M}_{1}\right)$-methyl methacrylate $\left(\mathrm{M}_{2}\right)$ system based on the values of mole fraction of three kinds of triad, namely, 121, 122 (and 221), and 222, which were evaluated from the relative intensities among corresponding three $\mathrm{OCH}_{3}$ peaks in its NMR spectrum. These ratios were insensitive to the feed ratios of monomers, slightly affected by the species of penultimate units, and considerably affected by the homogeneity of the system. These monomer reactivity ratios decreased with polymerization temperature. These conclusions could not be obtained if the monomer reactivity ratios were not determined from a single sample, because the estimated errors were comparable order of magnitude with the difference among experimental values in traditional methods.
\end{abstract}

KEY WORDS Monomer Reactivity Ratio / Copolymer / Poly(acrylonitrile-methyl methacrylate) / Penultimate Effect / Polymerization Temperature / NMR /

Mayo-Lewis theory ${ }^{1-4}$ is widely accepted as a tool to describe the propagation reaction in radical copolymerization using two parameters. However, there are some criticisms against this theory, i.e.,

1. The propagation reaction does not only depend on the species of the radical in the growing end and the adding monomer but also on those of preceding units in the growing chain. This effect is called penultimate unit effect.

2. The two parameters which are called monomer reactivity ratios are not universal constants decided by the species of radical and monomer, but they are affected by the environment such as polymerization temperature, solvent, polarity and so on.

3. Statistical nonstationarity and kinetic nonsteadiness must be considered in order to des-

* Present address: Katata Research Institute, Toyobo Co., Ltd., 1300-1 Hon-Katata, Otsu-shi 520-02, Japan. cribe the reaction.

The first criticism has been treated theoretically, ${ }^{5}$ and some experimental results have been also reported. For example, in the system of acrylonitrile $\left(\mathbf{M}_{1}\right)$ and methyl mathacrylate $\left(\mathbf{M}_{2}\right)$ four kinds of monomer reactivity ratios are given by $^{6}$

$$
\begin{array}{ll}
r_{11}=0.39, & r_{21}=0.20 \\
r_{12}=1.56, & r_{22}=1.01
\end{array}
$$

The second criticism has been studied experimentally by Lewis himself ${ }^{7}$ and he reported the independence of monomer reactivity ratios on polymerization temperature. However, in the system of styrene $\left(\mathbf{M}_{1}\right)$ and cinnamic acid $\left(\mathrm{M}_{2}\right)$ the relation

$$
r_{1}=(3.7 \pm 0.4) \exp (-(0.23 \pm 0.03) / T)
$$

has been reported. ${ }^{8}$ The last criticism has also been treated theoretically by Fueno and Furukawa. ${ }^{9}$ 
These criticisms were presented a few years after the original theory of Mayo and Lewis, but one can find only a few experimental studies on them. The main reason why these criticisms are not detected experimentally seems to lie on the method of estimating monomer reactivity ratios. Generally, the ratios are estimated by the plot of many data which are obtained from samples with different feed ratios. The Fineman - Ross method ${ }^{10}$ is a typical one. Such reactivity ratios obtained from many data include experimental errors which are inevitable. Of course, this error can be considerably reduced by averaging several data. This statement is true if the data used in the averaging are obtained under the same conditions. If the data used are obtained in different conditions such as with a difference in monomer feed ratios, then the other disadvantage occurs, namely, the dissipation of characteristics of the data due to the difference of condition. For example, if the difference in monomer reactivity ratios due to the difference in polymerization temperature is comparable with that due to the difference in monomer feed ratios, one cannot obtain the polymerization temperature dependence of monomer reactivity ratios by traditional methods. One must develop a new method to determine the monomer reactivity ratios from the data on a single sample.

The purpose of this paper is to report the derivation of the formulae to determine the monomer reactivity ratios in copolymerization reactions from a single sample and the application of these formulae to the system of acrylonitrile and methyl methacrylate.

\section{THEORETICAL}

If the mole fractions of dyads are given for the system of binary copolymerization of $M_{1}$ and $\mathrm{M}_{2}$, monomer reactivity ratios $r_{1}$ and $r_{2}$ can be determined form the values of these fractions in a single sample by the assumption of statistical stationarity. This is because there are three kinds of dyad fractions, namely, $\mathrm{I}(11)$, $\mathrm{I}(12)$ and $\mathrm{I}(22)$, and any two members of them are independent of the other by the normalization condition

$$
\mathrm{I}(11)+\mathrm{I}(12)+\mathrm{I}(22)=1
$$

The notation $\mathrm{I}(12)$ includes the fractions of 12 and 21-dyads because of the indistinguishability of these two dyads in dead polymers. The fact that the above number of independent variables is equal to the number of kinds of monomer reactivity ratios implies the possibility of the determination of monomer reactivity ratios from a single sample. Such a method to determine the monomer reactivity ratios has already been obtained by one (R.C.) ${ }^{11}$ of the present authors as

$$
\begin{aligned}
& r_{1}=\frac{\left[\mathrm{M}_{2}^{\circ}\right]}{\left[\mathrm{M}_{1}^{\circ}\right]} \frac{2 \mathrm{I}(11)}{(\mathrm{I} 12)} \\
& r_{2}=\frac{\left[\mathrm{M}_{1}^{\circ}\right]}{\left[\mathrm{M}_{2}^{\circ}\right]} \frac{2 \mathrm{I}(22)}{\mathrm{I}(12)}
\end{aligned}
$$

for the case of low conversion. In these equations $\left[\mathrm{M}_{1}{ }^{\circ}\right]$ and $\left[\mathrm{M}_{2}{ }^{\circ}\right]$ denote the molarities of initially fed monomers, $M_{1}$ and $M_{2}$. These formulae can naturally be extended to the formulae to determine the monomer reactivity ratios $r_{11}, r_{21}, r_{12}$, and $r_{22}$ from the mole fractions of triads in a single sample. They are as follows

$$
\begin{aligned}
& r_{11}=\frac{\left[\mathrm{M}_{2}^{\circ}\right]}{\left[\mathrm{M}_{1}^{\circ}\right]} \frac{2 \mathrm{I}(111)}{\mathrm{I}(112)} \\
& r_{21}=\frac{\left[\mathrm{M}_{2}^{\circ}\right]}{\left[\mathrm{M}_{1}^{\circ}\right]} \frac{\mathrm{I}(112)}{2 \mathrm{I}(212)} \\
& r_{12}=\frac{\left[\mathrm{M}_{1}^{\circ}\right]}{\left[\mathrm{M}_{2}^{\circ}\right]} \frac{\mathrm{I}(122)}{2 \mathrm{I}(121)} \\
& r_{22}=\frac{\left[\mathrm{M}_{1}^{\circ}\right]}{\left[\mathrm{M}_{2}^{\circ}\right]} \frac{2 \mathrm{I}(222)}{\mathrm{I}(122)}
\end{aligned}
$$

\section{EXPERIMENTAL}

Acrylonitrile of commercial grade was purified by washing with aqueous $\mathrm{NaOH}$ solution and with distilled water. After these processes it was dried with calcium chloride and with calcium hydride. The fraction boiling at $45 \sim 46^{\circ} \mathrm{C}$ $(250 \mathrm{~mm})$ was used as monomer, $\mathrm{M}_{1}$.

Methyl methacrylate of commercial grade was purified by successive washings with saturated aqueous $\mathrm{Na}_{2} \mathrm{~S}_{2} \mathrm{O}_{3}$ solution, with aqueous $\mathrm{NaOH}$ solution and with aqueous $\mathrm{NaCl}$ solution. It was dried with calcium chloride and with cal- 
cium hydride. The fraction boiling at $43 \sim$ $43.5^{\circ} \mathrm{C}(20 \mathrm{~mm})$ was used as monomer, $\mathrm{M}_{2}$.

Tetrahydrofuran was purified by the addition of $\mathrm{KOH}$ and of $\mathrm{CuCl}$, and distilled.

Benzoyl peroxide was recrystallized from the chloroform solution.

Copolymerization was carried out using $20 \mathrm{~g}$ of total monomers $\left(M_{1}\right.$ and $\left.M_{2}\right)$ in $25 \mathrm{ml}$ of solvent. Solvent used was tetrahydrofuran and $n$-hexane. The reaction was carried out in a sealed ampoule with $0.4 \mathrm{~mol} \%$ of initiator to total monomers. The reaction was stopped by methanol. After decantation the precipitate was successively washed by methanol. The conversion of all samples was less than $2.5 \%$. In the copolymerization at 80 and $100^{\circ} \mathrm{C}$ initiator was not used.

High-resolution nuclear magnetic resonance (NMR) of the samples obtained was measured in benzonitrile solution by JNM C-60H and JNM-PS 100 spectrometers at $130^{\circ} \mathrm{C}$.

\section{RESULTS AND DISCUSSION}

The region assigned to methoxyl protons in NMR spectra of acrylonitrile-methyl methacrylate copolymers splits into three peaks as shown in Figure 1. This phenomenon had already been found by Johnsen ${ }^{12}$ and Guillot. ${ }^{13}$ The latter concluded that this splitting did not originate from cotacticity, because of insensitivity of the splitting pattern to the operating frequency of the spectrometer.

Some of the methoxyl resonances in NMR spectra were reproduced in Figure 2. The peak centered at $3.86 \mathrm{ppm}$ from hexamethyl disiloxane used as a standard clearly grows with decrease of mole percent of fed monomer of methyl methacrylate, while the peak at $3.74 \mathrm{ppm}$ damps its relative intensity. Thus, one can safely assign these three peaks to $M_{1} M_{2} M_{1}, M_{1} M_{2} M_{2}$, and $M_{2} M_{2} M_{2}$ triads from the lower to upper fields. The location of the last peak coincides with the methoxyl peak in poly(methyl methacrylate).

Monomer reactivity ratios $r_{12}$ and $r_{22}$ are determined by the substitution of the quantities, $\mathrm{I}(121)$ and so on, by the relative intensities of the three peaks above mentioned.

Figure 3 is the plot of $r_{12}$ and $r_{22}$ against the feed ratio, $\left[\mathrm{M}_{1}^{\circ}\right] /\left[\mathrm{M}_{2}^{\circ}\right]$, in the homogeneous polymerization initiated by benzoyl peroxide in tetrahydrofuran at $40^{\circ} \mathrm{C}$. Both of $r_{12}$ and $r_{22}$ are independent of $\left[\mathrm{M}_{1}^{\circ}\right] /\left[\mathrm{M}_{2}{ }^{\circ}\right]$, and one has

$$
r_{12}=1.35 \pm 0.05, \quad r_{22}=1.28 \pm 0.01
$$

as the values of the monomer reactivity ratios in the system. These values do not coincide

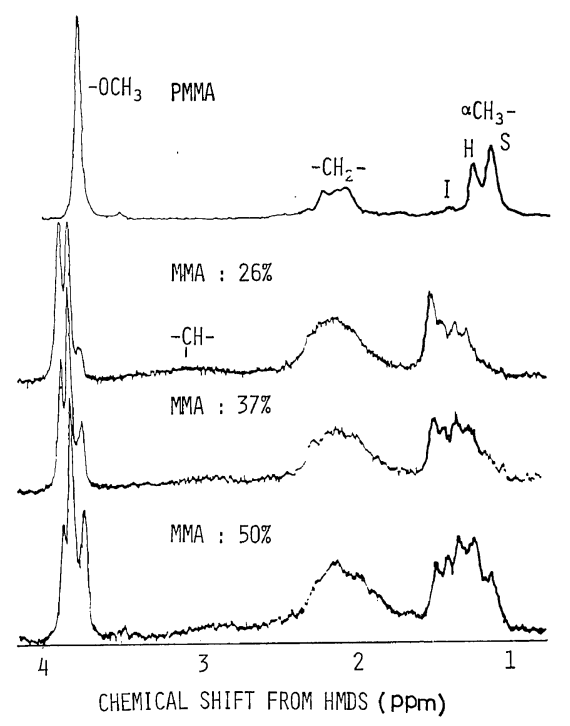

Figure 1. High resolution NMR spectra of poly (methyl methacrylate) and acrylonitrile-methyl methacrylate copolymer initiated by benzoyl peroxide in tetrahydrofuran at $40^{\circ} \mathrm{C}$.

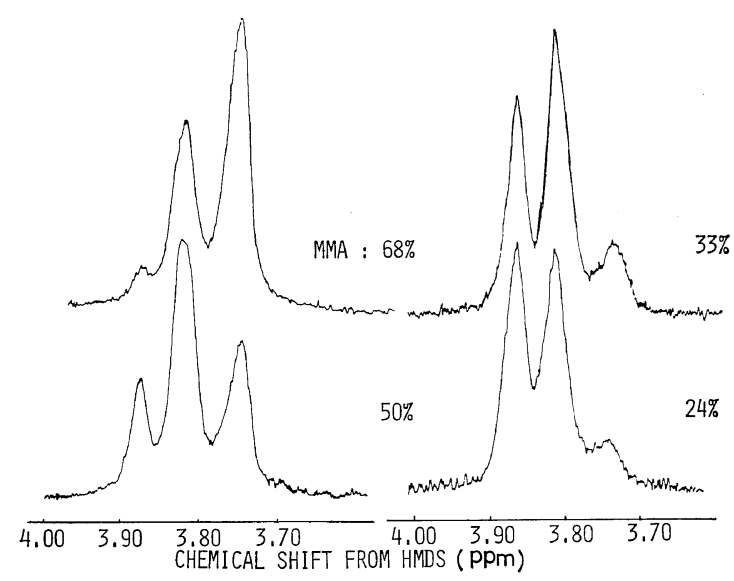

Figure 2. Methoxyl resonance in NMR spectra of acrylonitrile-methyl methacrylate copolymer initiated by benzoyl peroxide in tetrahydrofuran at $40^{\circ} \mathrm{C}$. 
with those in eq 1 . It can be concluded for the homogeneous system that monomer reactivity ratios, $r_{12}$ and $r_{22}$ are independent of feed ratios, and $r_{2}$ is slightly affected by the species of penultimate units.

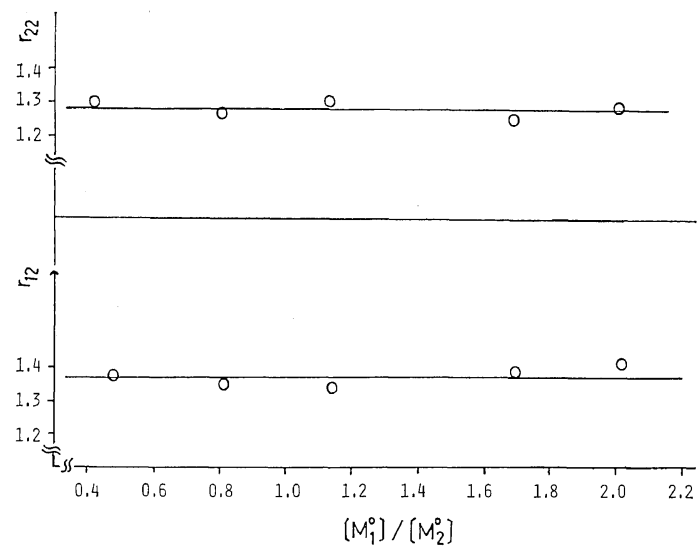

Figure 3. Dependence of $r_{12}$ and $r_{22}$ on $\left(\left[\mathrm{M}_{1}^{\circ}\right] /\right.$ $\left.\left[\mathrm{M}_{2}^{\circ}\right]\right)$ in the copolymerization of acrylonitrile $\left(\mathrm{M}_{1}\right)$ and methyl methacrylate $\left(\mathrm{M}_{2}\right)$ initiated by benzoyl peroxide in tetrahydrofuran at $40^{\circ} \mathrm{C}$.

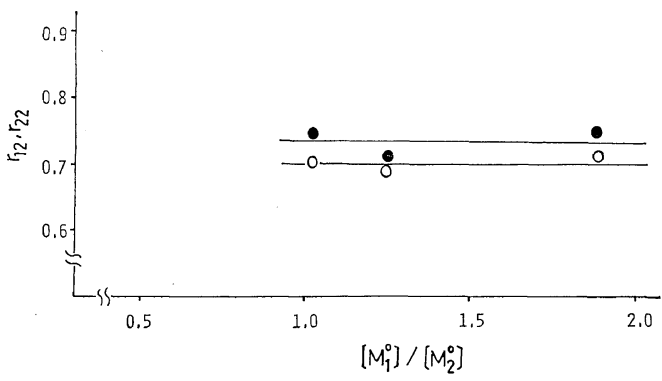

Figure 4. Dependence of $r_{12}(-)$ and $r_{22}(\bigcirc)$ on $\left(\left[\mathrm{M}_{1}{ }^{\circ}\right] /\left[\mathrm{M}_{2}{ }^{\circ}\right]\right)$ in the copolymerization of acrylonitrile $\left(\mathrm{M}_{1}\right)$ and methyl methacrylate $\left(\mathbf{M}_{2}\right)$ initiated by benzoyl peroxide in $n$-hexane at $40^{\circ} \mathrm{C}$.
A similar procedure was carried out for the samples by heterogeneous polymerization initiated by benzoyl peroxide in n-haxane at $40^{\circ} \mathrm{C}$, and one has Figure 4 and

$$
r_{12}=0.73 \pm 0.04, \quad r_{22}=0.70 \pm 0.03
$$

Figure 5 is the result of similar analysis in

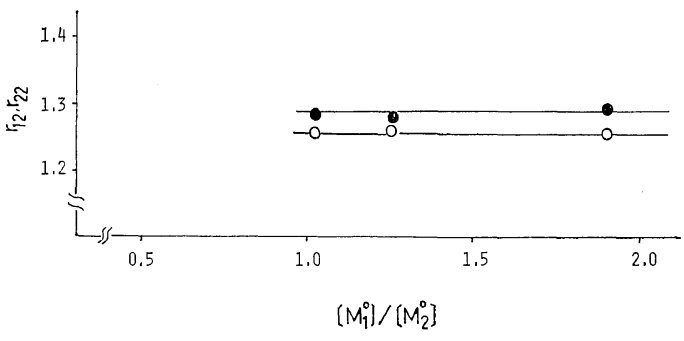

Figure 5. Dependence of $r_{12}(\bullet)$ and $r_{22}(\bigcirc)$ on $\left(\left[\mathrm{M}_{1}^{\circ}\right] /\left[\mathrm{M}_{2}^{\circ}\right]\right)$ in the bulk copolymerization of acrylonitrile $\left(\mathbf{M}_{1}\right)$ and methyl methacrylate $\left(\mathbf{M}_{2}\right)$ initiated by benzoyl peroxide at $40^{\circ} \mathrm{C}$.

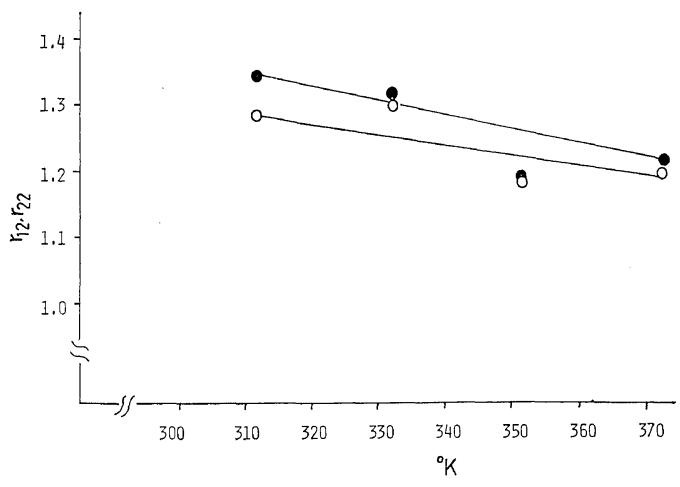

Figure 6. Dependence of $r_{12}(\boldsymbol{O})$ and $r_{22}(\bigcirc)$ on reaction temperature in the copolymerization of acrylonitrile $\left(\mathrm{M}_{1}\right)$ and methyl mathacrylate $\left(\mathrm{M}_{2}\right)$ at $\left[\mathrm{M}_{1}{ }^{\circ}\right] /\left[\mathrm{M}_{2}{ }^{\circ}\right] \doteqdot 1.26$ initiated by benzoyl peroxide (or initiated thermally in tetrahydrofuran.

Table I. Dependence of monomer reactivity ratios on polymerization temperature for the samples produced by homogeneous polymerization in tetrahydrofuran

\begin{tabular}{ccccccc}
\hline \multirow{2}{*}{$\begin{array}{c}\text { Polymerization } \\
\text { temp, }{ }^{\circ} \mathrm{C}\end{array}$} & $\begin{array}{c}\text { Feed ratio, } \\
{\left[\mathrm{M}_{1}{ }^{\circ}\right] /\left[\mathrm{M}_{2}{ }^{\circ}\right]}\end{array}$ & \multicolumn{3}{c}{ Triad fraction } & \multicolumn{2}{c}{ Reactivity ratios } \\
\cline { 5 - 7 } & $\mathrm{I}(121)$ & $\mathrm{I}(122)$ & $\mathrm{I}(222)$ & $r_{12}$ & $r_{22}$ \\
\hline 40 & 12.6 & 0.236 & 0.506 & 0.258 & 1.35 & 1.28 \\
60 & 1.26 & 0.241 & 0.501 & 0.258 & 1.31 & 1.30 \\
80 & 1.02 & 0.214 & 0.497 & 0.289 & 1.19 & 1.19 \\
100 & 1.26 & 0.260 & 0.503 & 0.243 & 1.22 & 1.21 \\
\hline
\end{tabular}


bulk polymerization initiated by benzoyl peroxide at $40^{\circ} \mathrm{C}$. In this case one has

$$
r_{12}=1.28 \pm 0.03, \quad r_{22}=1.26 \pm 0.01
$$

In all cases the monomer reactivity ratios, $r_{12}$ and $r_{22}$ were independent of feed ratios, and $r_{2}$ was slightly affected by the species of the penultimate units. However, monomer reactivity ratios were considerably affected by the homogeneity of system.

Figure 6 is the plot of $r_{12}$ and $r_{22}$ against the reaction temperature in copolymerizations corresponding to those in Figure 3. Monomer feed ratios were almost identical with each other as seen in Table I. Monomer reactivity ratios clearly decreased with reaction temperature.

\section{REFERENCES}

1. F. R. Mayo and F. M. Lewis, J. Amer. Chem. Soc. 66, 1954 (1944).
2. T. Alfrey, Jr. and G. Goldfinger, J. Chem. Phys., 12, 205 (1944).

3. F. T. Wall, J. Amer. Chem. Soc., 66, 2050 (1944).

4. I. Sakurada, "Kyojugo Hanno," Kobunshi Kagaku Kyokai, 1944, p 35.

5. E. Merz, T. Alfrey, Jr., and G. Goldfinger, J. Polym. Sci., 1, 75 (1946).

6. A. Guyot and J. Guillot, J. Macromol. Sci., Part A, 1, 793 (1967).

7. F. M. Lewis, C. Walling, W. Cummings, E. R. Briggs, and F. R. Mayo, J. Amer. Chem. Soc., 70, 1519 (1948).

8. C. A. Barson and M. S. Rizvi, Eur. Polym. J., 6, 241 (1970).

9. T. Fueno and J. Furukawa, J. Polym. Sci., Part A, 2, 3681 (1964).

10. M. Fineman and S. D. Ross, J. Polym. Sci., 5, 269 (1950).

11. R. Chûjô, J. Phys. Soc. Japan, 21, 2669 (1966).

12. U. Johnsen and K. Kolbe, Makromol. Chem., 116, 173 (1968).

13. J. Guillot, A. Guyot, and P.Q. Tho, J. Macromol. Sci., A2, 1303 (1968). 